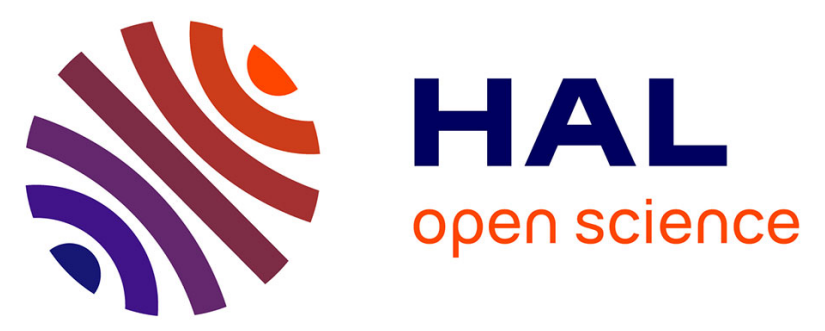

\title{
Enhancement of nanograting formation assisted by silver ions in a sodium gallophosphate glass
}

\author{
Marie Vangheluwe, Feng Liang, Yannick Petit, Patricia Hée, Yannick Ledemi, \\ Sébastien Thomas, Evelyne Fargin, Thierry Cardinal, Younes Messaddeq, \\ Lionel Canioni, et al.
}

\section{To cite this version:}

Marie Vangheluwe, Feng Liang, Yannick Petit, Patricia Hée, Yannick Ledemi, et al.. Enhancement of nanograting formation assisted by silver ions in a sodium gallophosphate glass. Optics Letters, 2014, 39 (19), pp.5491-5494. 10.1364/OL.39.005491 . hal-01074507

\section{HAL Id: hal-01074507 https://hal.science/hal-01074507}

Submitted on 23 Feb 2016

HAL is a multi-disciplinary open access archive for the deposit and dissemination of scientific research documents, whether they are published or not. The documents may come from teaching and research institutions in France or abroad, or from public or private research centers.
L'archive ouverte pluridisciplinaire HAL, est destinée au dépôt et à la diffusion de documents scientifiques de niveau recherche, publiés ou non, émanant des établissements d'enseignement et de recherche français ou étrangers, des laboratoires publics ou privés.

\section{(ㅇ)(1) $\$$}

Distributed under a Creative Commons Attribution - NonCommerciall 4.0 International 


\title{
Enhancement of nanograting formation assisted by silver ions in a sodium gallophosphate glass
}

\author{
Marie Vangheluwe, ${ }^{1,2}$ Feng Liang, ${ }^{2}$ Yannick Petit, ${ }^{1,3, *}$ Patricia Hée,,${ }^{2,3}$ Yannick Ledemi, ${ }^{2}$ Sébastien Thomas, ${ }^{3}$ \\ Evelyne Fargin, ${ }^{3}$ Thierry Cardinal, ${ }^{3}$ Younès Messaddeq, ${ }^{2}$ Lionel Canioni, ${ }^{1}$ and Réal Vallée ${ }^{2}$ \\ ${ }^{1}$ University of Bordeaux, LOMA, UMR 5798, F 33400 Talence, France and CNRS, LOMA, UMR 5798, F 33400 Talence, France \\ ${ }^{2}$ Centre d'Optique, Photonique et Laser Université Laval, 2375 Rue de la Terrasse, Québec, QC G1V oA6, Canada \\ ${ }^{3}$ CNRS, ICMCB, UPR 9048, F 33608 Pessac, France, and Univ. Bordeaux, ICMCB, UPR 9048, F 33400 Pessac, France \\ *Corresponding author: petit@icmcb bordeaux.cnrs.fr
}

\begin{abstract}
We investigated the influence of silver ions during the direct femtosecond laser induced formation of nanogratings (NGs) at the surface of a tailored sodium gallophosphate glass. We observed that the silver ions had a remarkable influence because the silver containing glass showed (1) lower fluence thresholds for the formation of the NGs; (2) much smoother NG shapes; and (3) a bifunctional behavior because fluorescence from laser induced silver clus ters occurs prior to the formation of the NGs. We demonstrate for the first time, to our knowledge, the formation of NGs assisted by noble metal ions, such as ions playing a kind of catalytic like role that enhances and improves the NG formation and its incubation process. Our innovative approach provides promising potential for further improvements in processes for NG formation.
\end{abstract}

OCIS codes: (160.2540) Fluorescent and luminescent materials; (140.3390) Laser materials processing; (140.3450) Laser induced chemistry; (190.4180) Multiphoton processes; (310.6628) Subwavelength structures, nanostructures.

Femtosecond (fs) laser manufacturing has led to continuous innovation in the last decade, especially in the inscription of structures with inner dimensions smaller than those of the considered beam size, such as the formation of nanogratings (NGs), which shows great potential for optical waveguides, solar cells, and biological devices [1 3]. The spatial distributions of NGs in bulk silica glass have been reported in detail in pioneer and recent works [ $\underline{4}$ 7], both for bulk and surface NGs. Different mechanisms and descriptions have been proposed, including the incubation model [8]. While NGs have mostly been studied in silica by precisely tuning the laser irradiation parameters, very little attention has been paid to the material itself until now. No effort has been clearly made to adapt the considered glass matrices, either by exploring exotic compositions or by engineering a photosensitized material with the introduction of noble metal ions [9]. While silica cannot stand large amounts of silver ions [10], phosphate glasses can sustain high concentrations, allowing the direct laser writing (DLW) of strong and stable linear and nonlinear optical properties, such as silver cluster fluorescence, third-harmonic generation, or electric field-induced second-harmonic generation [11 16]. In this framework, the photochemistry of silver ions triggered under fs laser irradiation has never been explored for NG formation.

In this Letter, we study the role of silver ions incorporated in a tailored sodium gallophosphate matrix during the NG formation at its surface by means of fs laser irradiation. Accordingly, we compare the behaviors of such a sodium gallophosphate glass matrix with and without silver ions. In the silver-containing glass, we observed significant improvements due to the silver ions, such as lower fluence thresholds for the NG formations and much smoother NG shapes (also much smoother than the usual nanostructures, as in fused silica [17]). We also observed that fluorescent silver clusters are created prior to NG formation at lower fluencies, leading to spatially correlated fluorescent patterns and NGs. Moreover, material removal was eased in the case of the silver-containing glass, with cleaner final NGs. Our innovative approach, based on noble metal doping, creates the potential for the optimal engineering and generation of NGs.

Glass samples with the initial composition $20.0 \mathrm{Ga}_{2} \mathrm{O}_{3}$ $40.0 \mathrm{Na}_{2} \mathrm{O}-40.0 \mathrm{P}_{2} \mathrm{O}_{5}(\mathrm{~mol} . \%)$ and $97\left[20.0 \mathrm{Ga}_{2} \mathrm{O}_{3}-40.0 \mathrm{Na}_{2} \mathrm{O}-\right.$ $\left.40.0 \mathrm{P}_{2} \mathrm{O}_{5}\right]-3 \mathrm{Ag}_{2} \mathrm{O}$ (mol. \%), respectively labeled GPN and GPN-Ag, were synthesized using the conventional meltquenching technique. The proper amount of $\mathrm{Ga}_{2} \mathrm{O}_{3}$, $\mathrm{NaPO}_{3}$, and $\mathrm{Ag}_{2} \mathrm{O}$ powders were thoroughly mixed, placed in a $100 \%$ Pt platinum crucible, heated up to $1100^{\circ} \mathrm{C}$ in ambient air, and kept at maximum temperature for $30 \mathrm{~min}$ before quenching. The glass samples obtained were annealed for $6 \mathrm{~h}$ at $40^{\circ} \mathrm{C}$ below the glass transition temperature of each sample. Further description of the preparation and characterizations of the sodium gallophosphate glasses can be found in [18]. The GPN and GPN-Ag samples were cut into pieces with $1 \mathrm{~mm}$ thickness. Finally, one of each were bound together to a silica glass substrate and polished to optical quality. This allows the two material samples to share the same interface, which guaranteed identical focusing conditions during laser structuring.

The experimental setup of the NG formation, as detailed elsewhere [8], is composed of a commercial Ti:Sapphire chirped-pulse amplification laser system (Spitfire, Spectra-Physics) with a maximum output energy of $2 \mathrm{~mJ}$ per pulse at the central wavelength $\lambda_{\text {laser }}=800 \mathrm{~nm}$, with a pulse duration $\tau=45 \mathrm{fs}$ (FWHM) and a repetition rate $f_{\text {rep }}=1 \mathrm{kHz}$. The beam was tightly focused at the surface sample with a microscope objective (Mitutoyo, $50 \times$, N.A. $=0.42$ ), which was adjusted by maximizing the retro-reflection from a He Ne laser beam. The diameter of the focal spot was estimated at $2 \times \omega_{0}=2 \mu \mathrm{m}$. The samples were mounted on motorized threedimensional (3D) translation stages (Melles Griot). The 
considered writing speeds, with sample motion at constant speed from $v=10$ to $100 \mu \mathrm{m} / \mathrm{s}$, allowed the estimation of the number of pulses $N$ that overlapped at each position during laser structuring, as $N=$ $2 \times \omega_{\mathrm{o}} \times f_{\text {rep }} / v$. The laser beam was linearly polarized perpendicular to the sample translation direction. The energy per pulse ( $E_{p}=10$ to $100 \mathrm{~nJ}$ ) was adjusted with a half-wave plate and a Glan polarizer and was measured after the objective lens. The estimated pulse fluence is $F_{p}=E_{p} / \pi \times \omega_{0}^{2}$, whereas the effective beam fluence is defined as $F=N \times F_{p}$. After irradiation, the samples were imaged first by standard bright-field and fluorescence microscopy. Then, the samples were gently cleaned in an ultrasonic bath with distilled water for 4 min and characterized by a scanning electron microscope (SEM in the secondary electron emission mode) (FEI, model Quanta 3D FEG).

Both the silver-free and silver-containing samples were laser-processed and subsequently characterized under the same experimental conditions to ensure that the differences between GPN and GPN-Ag can only be attributed to the presence of silver ions.

The refractive indices at $\lambda_{\text {laser }}$ of the GPN and GPN-Ag samples were estimated at 1.536 and 1.544 , respectively, as inferred from the Sellmeier fitting of M-lines prism coupling measurements (Metricon 2010) at different wavelengths (532, 633, 972, 1308, and $1538 \mathrm{~nm}$ ). The close values of the refractive indices of both the Agdoped and nondoped GPN samples ensure that the focusing conditions will be similar for both glasses. Optical transmission spectroscopy of the two pristine glasses led to the estimation of the linear absorption coefficient in the UV-visible range. As seen in Fig. 1(a), the transmission cutoff of the GPN-Ag is significantly shifted toward longer wavelengths, suggesting a potentially much larger nonlinear three-photon absorption cross section (at $266 \mathrm{~nm}$ ) for the fs irradiation [17]. Fluorescence spectroscopy revealed the silver-doping influence because the pristine GPN-Ag sample showed a strong excitation band centered at $272 \mathrm{~nm}$, related to a broad emission band centered at 412 nm, as shown in Fig. 1(b). Such fluorescence behavior is typical of silver ion-containing glasses [19], whereas the nondoped sample showed no fluorescence

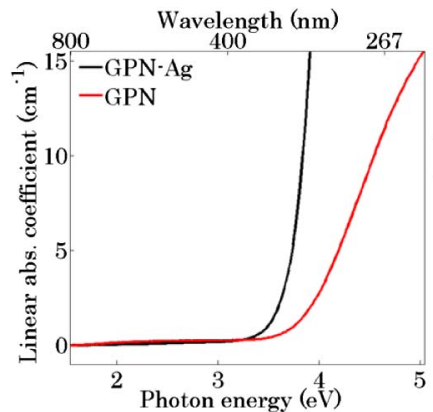

(a)

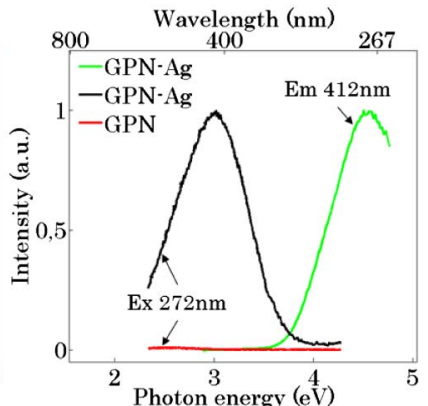

(b)
Fig. 1. (a) Linear absorption coefficients of the GPN and GPN Ag pristine samples and the upshifted UV cutoff wavelength due to the $\mathrm{Ag}^{+}$insertion. (b) Normalized excitation (emission wave length at $412 \mathrm{~nm}$ ) and emission fluorescence (excitation wave length at $272 \mathrm{~nm}$ ) spectra of the pristine GPN Ag glass. Absence of emission in the nondoped GPN sample confirms that these fluorescent properties result from the silver ions. response. The excitation band of silver ions is to be correlated with the shift of the transmission cutoff in the GPN-Ag, strongly suggesting that the introduction of such ions is responsible for enhanced nonlinear absorption during the laser structuring process.

Laser irradiations were first performed at constant speed $(20 \mu \mathrm{m} / \mathrm{s})$ by increasing the pulse energy from 10 to 65 nJ. Features were observed under bright-field microscopy and luminescence imaging (excitation by a laser diode at $483 \pm 35 \mathrm{~nm}$ and collection after a bandpass filter at $536 \pm 40 \mathrm{~nm}$ ). The GPN sample showed a damage threshold at $3540 \mathrm{~nJ}$ per pulse with bright-field microscopy [Fig. 2(a)], but it showed no sign of a fluorescence response [Fig. 2(c)]. Comparatively, the GPN-Ag sample showed a much lower threshold, with optical damages starting from $2530 \mathrm{~nJ}$ [Fig. 2(b)]. Additionally, GPN-Ag led to a fluorescent emission [Fig. 2(d)], which is typical of the laser-induced creation of silver clusters [12], with an increasing fluorescence emission while increasing the input pulse energies. Probing the associated migration of silver ions during the creation of silver clusters is still in progress. Fluorescent clusters appeared prior to the damage threshold in GPN-Ag, demonstrating the photoreactivity of the silver ions in the early stages of the glass matrix modifications toward NG formation. Finally, no evidence of metallic silver nanoparticle formation was observed under such laser irradiances, suggesting their absence in these experimental conditions.

Once irradiated and softly cleaned with distilled water, the revealed structures were then observed with SEM, showing a NG formation threshold between 25 and $30 \mathrm{~nJ}$ per pulse in GPN-Ag (Fig. 3), which is significantly lower than the $3540 \mathrm{~nJ}$ required in the nondoped GPN sample (Fig. 3). In addition to the lowering of the NG formation threshold, the SEM images also revealed that the NGs in GPN-Ag were impressively smoother than those in GPN. Their full width-labeled W-is also wider for given irradiation conditions, including a larger number of grooves. The width of individual grooves typically

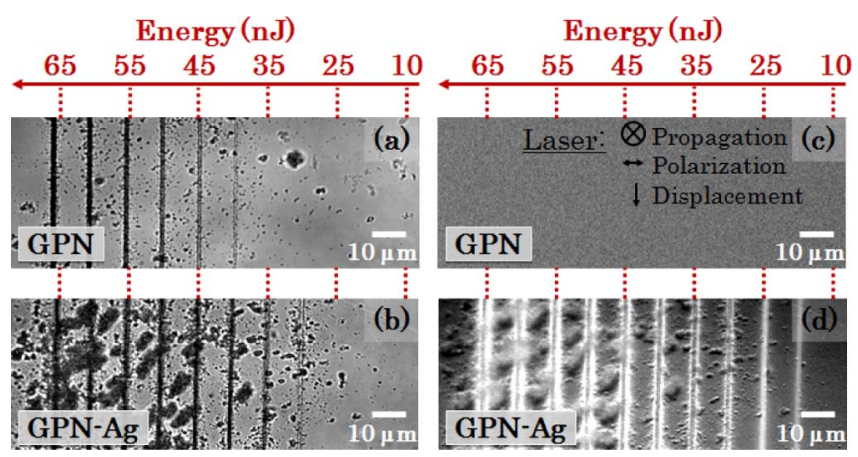

Fig. 2. (a) and (b) Bright field images $(100 \times, \mathrm{NA}=0.9)$ of the GPN and GPN Ag samples, respectively, after the femtosecond (fs) direct laser writing (DLW) of lines at the surface at a con stant scan speed at $20 \mu \mathrm{m} / \mathrm{s}$ for decreasing pulse energies be fore the cleaning process of the samples. (c) and (d) Related fluorescence images excited by a laser diode at $483 \pm 35 \mathrm{~nm}$ and collected after a bandpass filter at $536 \pm 40 \mathrm{~nm}$. These im ages clearly indicate that (1) NGs in GPN Ag appear for lower pulse energies than those in GPN; and (2) silver cluster fluores cence patterns appear prior to NG formation in GPN Ag. 


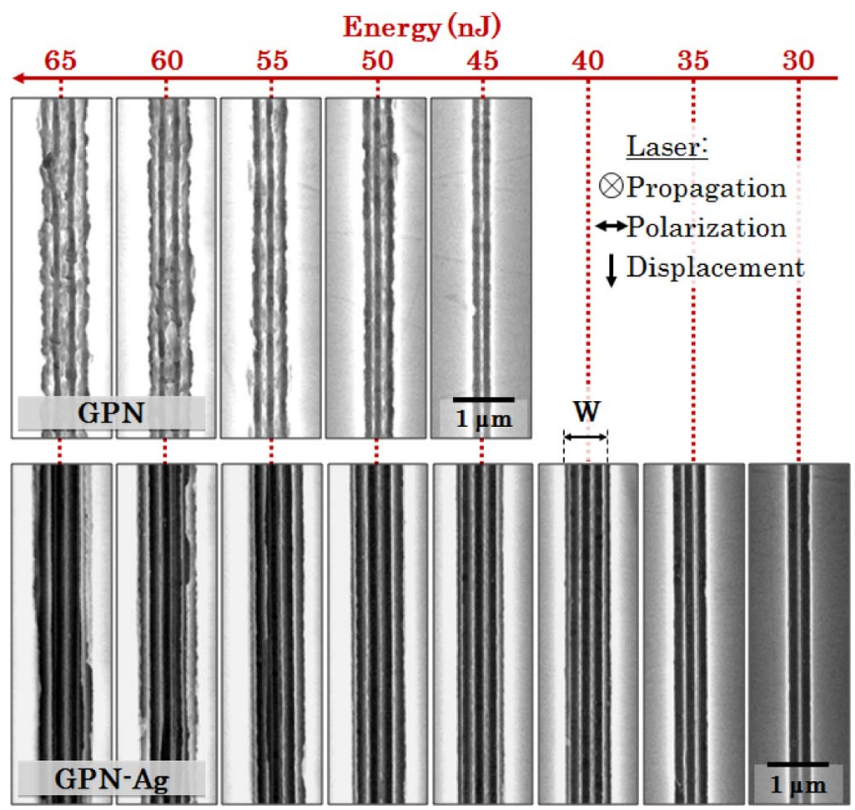

Fig. 3. Scanning electron microscope (SEM) images (secon dary electron emission mode) of the femtosecond (fs) laser induced linear structures above the damage threshold for both the GPN and GPN Ag irradiated samples.

spans from 44 to $290 \mathrm{~nm}$ for GPN-Ag compared to 146 to $204 \mathrm{~nm}$ for GPN (Fig. 3). These observations, especially the lower limit of $44 \mathrm{~nm}$ in GPN-Ag (as qualitatively visible at the edges of the NGs for 40, 45, and $50 \mathrm{~nJ}$ per pulse), illustrate the different dynamics of both the ionization rates and incubation processes observed in the two samples-such aspects directly influencing the morphology of the NGs, as evidenced in Fig. $\underline{4}$. The optical damage threshold increases with the scan speed of the sample, with higher thresholds for the nondoped sample [Fig. 4(a)]. Moreover, NGs in GPN-Ag showed a larger width and number of grooves compared to those in GPN, which were assessed from the SEM images, leading to distinct typical square root dependences versus pulse energies [Fig. 4(b)] in agreement with the incubation dynamics proposed elsewhere [8,20]. Further optical characterization of the diffraction efficiencies of the NGs will require additional work with larger structured areas.
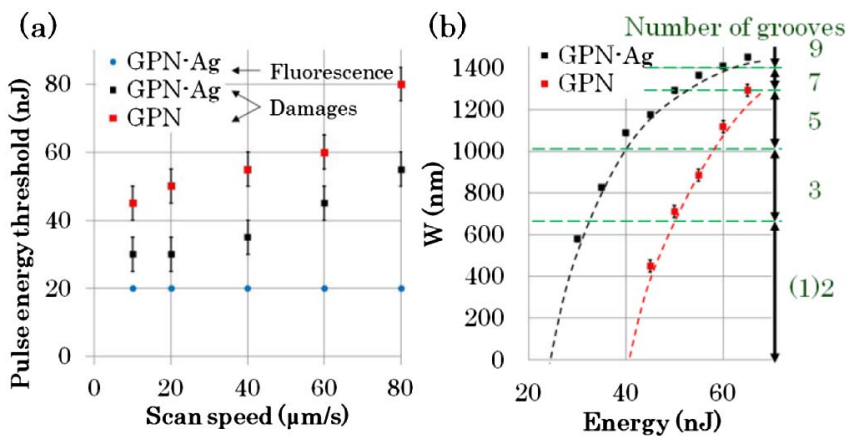

Fig. 4. (a) Pulse energies for the optical damage threshold ver sus the scan speed in the GPN and GPN Ag samples, as well as the fluorescence threshold for the latter. (b) Compared incuba tion effects related to the width $\mathrm{W}$ and the corresponding number of grooves for a scan speed of $20 \mu \mathrm{m} / \mathrm{s}$.
The preceding results can be interpreted as follows. First, the pulse energy threshold for optical damage grows more or less linearly with scan speed, which is the signature of a beam fluence-dependent process. On the contrary, the fluorescence threshold is independent of scan speed and solely depends on pulse energy, which is related to the beam intensity. The insertion of a doping species (the silver ions herein) provides a higher ability to release free electrons from nonlinear absorption under fs irradiation as compared to the glass matrix itself. In this sense, the silver ion population behaves as an extra reservoir of accessible electrons, more efficiently released than those from the glass matrix. Once released into the glass matrix, this larger amount of electrons can more efficiently trigger the incubation process via the enhanced creation of defects, which explains the lowering of the NG formation threshold in GPN-Ag. Moreover, ionizing silver ions is much less destabilizing for the glass network than ejecting electrons from the glass matrix itself, which corroborates the much smoother NG structures in the doped glass. Through this approach, the initial ionization step is better controlled and, somehow, the initial homogeneous distribution of silver ions makes this first step less affected by random variations in the nondoped material. Then, in the second step, the creation of fluorescent silver clusters indicates that silver also undergoes reduction processes. Because $\mathrm{Ag}^{+}$ions initially behave as potential electron donors, whereas silver clusters behave as electron acceptors, silver species globally act as a kind of catalytic-like actor during the laser structuring and the NG formation, even though silver globally undergoes irreversible reactions, with the production of new and stable silver clusters. Therefore, silver ions play an active role in the series of phenomena that occur during NG formation, most likely by providing additional free electrons and defects but also by making the series of phenomena free from the stochastic distributions of defects in the nondoped material. These considerations lead to the enhancement of NG formation with larger grooves but also to significantly improved quality and smoothness. Such an innovative approach could be extended to some other elements with the potential ability to act as homogeneously distributed defects inserted in a glassy matrix, as far as such doping species show a sufficiently high solubility in the selected glass system. To better understand the silver-mediated processes, further studies should also investigate ionic motions (for instance, the potential migration of silver ions).

In this Letter, we have reported on NGs obtained in a new phosphate glass composition. We have presented the first demonstration (to our knowledge) of the silver ion-mediated formation of NGs, which led to a significant lowering of the NG formation threshold, wider structures with a larger number of grooves correlated to silver cluster fluorescence properties, and a remarkable improvement of NG smoothness. By providing a reservoir of available free electrons, silver ions appear as electron donors that also allow overcoming the stochastic distribution of defects in the glassy matrix, enhancing the NG formation. Such a very general approach (restricted to the specific use of silver ions herein) should further 
provide enhanced abilities for the manufacturing of highquality NGs for photonic devices.

This research was supported by the French Agency of National Research, the French Aquitaine region, the Cluster of Excellence LAPHIA, the Canada Foundation for Innovation (CFI), the Canadian Excellence Research Chair (CERC) in Photonic Innovations, the Natural Sciences and Engineering Research Council of Canada (NSERC), and the Fonds de Recherche du Québec-Nature et Technologies (FRQNT). We thank S. Gagnon and M. D'Auteuil for their technical support.

\section{References}

1. F. Liang, R. Vallée, and S. L. Chin, Opt. Express 20, 4389 (2012).

2. C. Mauclair, A. Mermillod Blondin, N. Huot, E. Audouard, and R. Stoian, Opt. Express 16, 5481 (2008).

3. F. Zhang, H. Zhang, G. Dong, and J. Qiu, J. Opt. Soc. Am. B 31, 860 (2014).

4. P. G. Kazansky, H. Inouye, T. Mitsuyu, K. Miura, J. Qiu, and K. Hirao, Phys. Rev. Lett. 82, 2199 (1999).

5. Y. Shimotsuma, P. G. Kazansky, J. Qiu, and K. Hirao, Phys. Rev. Lett. 91, 247405 (2003).

6. R. Taylor, C. Hnatovsky, and E. Simova, Laser Photon. Rev. 2, 26 (2008).

7. S. Richter, A. Plech, M. Steinert, M. Heinrich, S. Döring, F. Zimmermann, U. Peschel, E. B. Kley, A. Tünnermann, and S. Nolte, Laser Photon. Rev. 6, 787 (2012).

8. F. Liang, R. Vallée, D. Gingras, and S. L. Chin, Opt. Mater. Express 1, 1244 (2011).
9. A. Royon, Y. Petit, G. Papon, M. Richardson, and L. Canioni, Opt. Mater. Express 1, 866 (2011).

10. M. Yamane and Y. Asahara, Glass for Photonics (Cambridge University, 2000).

11. K. Bourhis, A. Royon, M. Bellec, J. Choi, A. Fargues, M. Treguer, J. J. Videau, D. Talaga, M. Richardson, T. Cardinal, and L. Canioni, J. Non Cryst. Solids 356, 2658 (2010).

12. M. Bellec, A. Royon, K. Bourhis, J. Choi, B. Bousquet, M. Treguer, T. Cardinal, J. J. Videau, M. Richardson, and L. Canioni, J. Phys. Chem. C 114, 15584 (2010).

13. A. Royon, K. Bourhis, M. Bellec, G. Papon, B. Bousquet, Y. Deshayes, T. Cardinal, and L. Canioni, Adv. Mater. 22, 5282 (2010).

14. L. Canioni, M. Bellec, A. Royon, B. Bousquet, and T. Cardinal, Opt. Lett. 33, 360 (2008).

15. G. Papon, Y. Petit, N. Marquestaut, A. Royon, M. Dussauze, V. Rodriguez, T. Cardinal, and L. Canioni, Opt. Mater. Ex press 3, 1855 (2013).

16. G. Papon, N. Marquestaut, Y. Petit, A. Royon, M. Dussauze, V. Rodriguez, T. Cardinal, and L. Canioni, J. Appl. Phys. 115, 113103 (2014).

17. F. Liang, "Femtosecond laser writing of nanogratings on the surface of fused silica," Ph.D. thesis (Université Laval, 2012).

18. P. Hee, R. Christensen, Y. Ledemi, J. C. Wren, M. Dussauze, T. Cardinal, E. Fargin, S. Kroeker, and Y. Messaddeq, J. Mater. Chem. C 2, 7906 (2014).

19. I. Belharouak, C. Parent, B. Tanguy, G. Le Flem, and M. Couzi, J. Non Cryst. Solids 244, 238 (1999).

20. F. Liang, R. Vallée, and S. L. Chin, Appl. Phys. Lett. 100, 251105 (2012). 